\title{
Local drug delivery system for the treatment of tongue squamous cell carcinoma in rats
}

\author{
MARIAM Z. KAKABADZE ${ }^{1}$, TEONA PARESISHVILI ${ }^{2}$, KONSTANTINE MARDALEISHVILI ${ }^{3}$, \\ ZURAB VADACHKORIA ${ }^{4}$, NICHOLAS KIPSHIDZE ${ }^{5}$, MIKHEIL JANGAVADZE $^{1}$, LIA KARALASHVILI ${ }^{2}$, \\ KETEVAN GHAMBASHIDZE ${ }^{2}$, DAVID CHAKHUNASHVILI ${ }^{2}$ and ZURAB KAKABADZE $^{2}$ \\ ${ }^{1}$ Department of Clinical Anatomy and Operative Surgery, Iv. Javakhishvili Tbilisi State University, 0179 Tbilisi; \\ ${ }^{2}$ Department of Clinical Anatomy, Tbilisi State Medical University, 0186 Tbilisi; \\ ${ }^{3}$ Department of Head and Neck Surgery, Mardaleishvili Medical Center, 0179 Tbilisi; \\ ${ }^{4}$ Department of Child and Adolescent Maxillo-facial Surgery and Surgical Stomatology, \\ Tbilisi State Medical University, 0186 Tbilisi, Georgia; ${ }^{5}$ Department of Interventional Cardiology, \\ Cardiovascular Research Foundation, New York, NY 10019, USA
}

Received August 2, 2021; Accepted October 11, 2021

DOI: $10.3892 / \mathrm{ol} .2021 .13131$

\begin{abstract}
The present study describes a local drug delivery system with two functions, which can suppress tumor growth and accelerate wound healing. The system consists of a two-layer multicomponent fibrin-based gel (MCPFTG). The internal layer of MCPFTG, which is in direct contact with the wound surface, contains cisplatin placed on a CultiSpher-S collagen microcarrier. The external layer of MCPFTG consists of a CultiSpher-S microcarrier with lyophilized bone marrow stem cells (BMSCs). The efficacy of MCPFTG was evaluated in a rat model of squamous cell carcinoma of the tongue created with 4-nitroquinoline 1-oxide. The results of the study showed that, within 20-25 days, a non-healing wound of the tongue was formed in animals that underwent only $85 \%$ resection of squamous cell carcinoma, while rapid progression of the residual tumor was concomitantly observed. Immunohistochemical methods revealed high expression of cyclin D1 and low expression of E-cadherin in these animals. Additionally, high expression of p63 and Ki-67 was noted. In $80 \%$ of animals with squamous cell carcinoma of the tongue that were treated with MCPFTG after $85 \%$ tumor resection, a noticeable suppression of tumor growth was evident throughout 150 days, and tumor recurrence was not detected. Immunohistochemistry revealed
\end{abstract}

Correspondence to: Dr Teona Paresishvili, Department of Clinical Anatomy, Tbilisi State Medical University, 33 Vazha-Pshavela Avenue, 0186 Tbilisi, Georgia

E-mail: t.paresishvili@gmail.com

Abbreviations: 4NQO, 4-nitroquinoline 1-oxide; PEG, polyethylene glycol; PGF, pegylated fibrinogen; CPtCS, cisplatin-loaded CultiSpher-S; BMSC, bone marrow stem cell

Key words: oral squamous cell carcinoma, cisplatin, local drug delivery system, fibrin gel, bone marrow stem cell low or moderate expression of cyclin D1, and high expression of E-cadherin throughout the whole observation period. The MCPFTG-based local drug delivery system was shown to be effective in suppressing tumor growth and preventing recurrence.MCPFTG decreased the toxicity of cisplatin and enhanced its antitumor activity. In addition, lyophilized paracrine BMSC factors present in MCPFTG accelerated wound healing after tumor removal. Thus, the present study suggests novel opportunities for the development of a multifunctional drug delivery system for the treatment of squamous cell carcinoma.

\section{Introduction}

According to global cancer statistics, the incidence of oral cancer is $>300,000$ cases and it causes 145,400 cancer-associated deaths annually (1). The most widespread malignant tumor of the mouth is squamous cell carcinoma, which occurs in $80-90 \%$ of all oral cancer cases (2). Currently, the treatment methods of squamous cell carcinoma include surgery, chemotherapy, radiation therapy, immunotherapy and targeted therapy. These treatment methods have their respective applications and roles in the treatment process. Surgical resection is a key treatment method for tumors of the oral cavity and oropharynx (3-5). However, as noted by various studies, after tumor resection, the presence of residual tumor cells is frequent, which usually leads to tumor recurrence (6-8). The aim of the present study was not to discuss the preferences regarding the selection of surgical methods of oral squamous cell carcinoma treatment, nor the role of surgical margins, tumor location, size and depth, and histological differentiation, since these aspects have already been described by multiple studies in detail (9-11). The present study focused instead on controlled drug delivery systems. It was hypothesized that, following tumor resection, a gel-based targeted drug delivery system would prevent metastasis formation. For this purpose, a biocompatible two-layer multicomponent fibrin-based gel (MCPFTG) was developed. The efficacy of MCPFTG was 
assessed in a rat model of squamous cell carcinoma of the tongue constructed using 4-nitroquinoline 1-oxide (4NQO).

\section{Materials and methods}

Creation of the multicomponent gel. A multicomponent gel was created by slightly modifying a previously published protocol for creating polyethylene glycol (PEG)-fibrin gel, which was developed by Zhang et al (12). TBS (40 mg/ml; pH 7.8) and $250 \mu$ PEG were added to $500 \mu 1$ commercially purchased fibrinogen (MilliporeSigma). The acquired compound was then placed in a Falcon ${ }^{\circledR}$ 24-well Companion Plate (Falcon; Corning Life Sciences) and incubated at $37^{\circ} \mathrm{C}$ with $5 \% \mathrm{CO}_{2}$ and $90 \%$ humidity for $20 \mathrm{~min}$. The compound that was generated through this method was called pegylated fibrinogen (PGF).

Preparation of cisplatin-loaded CultiSpher-S (CPtCS) microcarrier. A total of $0.1 \mathrm{~g}$ commercially purchased microcarrier CultiSpher-S (MilliporeSigma) was placed in $5.0 \mathrm{ml}$ cisplatin solution (KOÇAK Farma), and $3.0 \mathrm{ml} 1 \%$ sodium citrate was added. The solution was then placed in a spinner flask, and stirred at $200 \mathrm{xg}$ for $4 \mathrm{~h}$ at room temperature. Subsequently, the solution was transferred to a 50-ml Falcon test tube (Falcon; Corning Life Sciences), where $15 \mathrm{ml}$ cisplatin solution was added, and subsequently centrifuged at a $10,000 \mathrm{x}$ g for $10 \mathrm{~min}$ at $40^{\circ} \mathrm{C}$. After removing the supernatant, $\mathrm{CPtCS}$ was placed in a freeze dryer (Power Dry PL 6000 Freeze Dryer; Heto Lab Equipment). At first, the shelf temperature was set at $-32^{\circ} \mathrm{C}$, and the vacuum was controlled under $10 \mathrm{~Pa}$. The drying process lasted for $16 \mathrm{~h}$. The shelf was then heated up to $20^{\circ} \mathrm{C}$ at a rate of $0.2^{\circ} \mathrm{C} / \mathrm{min}$ and held for $6 \mathrm{~h}$. When the process was completed, CPtCS was stored under sterile conditions at room temperature until further use.

Preparation of bone marrow stem cell (BMSC)-loaded CultiSpher-S microcarrier. BMSCs were acquired as previously described (13). Briefly, 20 Lewis inbred laboratory rats of both sexes weighing 200-250 g were used in the present study. Animals were euthanized with thiopental sodium (MilliporeSigma) with an intraperitoneal dose of $200 \mathrm{mg} / \mathrm{kg}$. The lower extremities were amputated after being treated with $70 \%$ ethanol solution. Femurs, which were cleared of muscle tissues, were resected in the epiphysis and diaphysis areas. A needle was inserted into the lumen of the bone canal, and a syringe was used to elute the bone marrow with DMEM (MilliporeSigma). The mononuclear fraction was isolated by density gradient centrifugation at $400 \mathrm{xg}$ for $30 \mathrm{~min}$ at room temperature using Ficoll Paque Plus or Ficoll Paque Premium solution (GE Healthcare Bio-Sciences). After being washed with PBS, the cells were centrifuged at $200 \mathrm{x}$ g at $24^{\circ} \mathrm{C}$ for $5 \mathrm{~min}$. Together with $0.1 \mathrm{~g}$ microcarrier CultiSpher-S, the acquired stem cells were placed in 12 of the wells of a Falcon 24-well Companion Plate (Falcon; Corning Life Sciences), and incubated at $37^{\circ} \mathrm{C}$ with $5 \% \mathrm{CO}_{2}$ and $90 \%$ humidity for $1 \mathrm{~h}$. DMEM, 10\% FBS (MilliporeSigma), $50 \mathrm{U} / \mathrm{ml}$ penicillin and $0.05 \mathrm{mg} / \mathrm{ml}$ streptomycin was used as the cell culture medium. An orbital shaker (110 rpm), onto which a plate was placed, was then introduced into the incubator. The cells were co-cultured with CultiSpher-S for 7 days. The culture medium was changed every 3 days. Upon culture, CultiSpher-S and BMSCs were placed in a freeze drier for lyophilization, and then stored under sterile conditions at a room temperature until further use.
For creating an external layer of the gel, $500 \mu \mathrm{l}$ fibrinogen, $250 \mu \mathrm{l}$ PEG, $0.1 \mathrm{~g}$ microcarrier CultiSpher-S and $0.5 \mathrm{mg}$ cisplatin were used. For the creation of the internal layer, $500 \mu \mathrm{l}$ fibrinogen, $250 \mu 1$ PEG, $0.1 \mathrm{~g}$ microcarrier CultiSpher-S and $2.5 \times 10^{6}$ freeze-dried BMSCs were used.

Rat model of squamous cell carcinoma of the tongue. A total of 60 male specific pathogen-free Lewis laboratory rats, (age, 8 weeks; weight, $\sim 250 \mathrm{~g}$ ), were used for establishing a model of squamous cell carcinoma of the tongue. The animals were acquired from the vivarium of Tbilisi State Medical University (Tbilisi, Georgia). The rats were maintained under controlled conditions at $24 \pm 2^{\circ} \mathrm{C}$ using a 12 -h light-dark cycle and with free access to water and food. The studies were performed in accordance with the recommendations of the Animal Care and Use Committee of Tbilisi State Medical University. All animal protocols were approved by a recognized institutional review board (Tbilisi State Medical University; approval no. 812).

For establishing a model of squamous cell carcinoma of the tongue in rats, $4 \mathrm{NQO}$ powder (MilliporeSigma) was used, which was dissolved in the drinking water of the animals to a final concentration of $0.02 \mathrm{~g} / \mathrm{l}(20 \mathrm{ppm})$. The water was changed on a weekly basis (14). Notably, 4NQO is widely used to study oral carcinogenesis in animals $(15,16)$, as it can lead to induction of all phases of oral carcinogenesis (including hyperplasia, dysplasia, acute dysplasia, carcinoma in situ and squamous cell carcinoma).

Methods of treatment of squamous cell carcinoma of the tongue in rats. After successful modeling, the 60 rats were divided into three groups: Group I, 4-NQO untreated controls $(\mathrm{n}=20)$; group II, 4-NQO + surgery $(\mathrm{n}=20)$; and group III, $4-\mathrm{NQO}+$ surgery + MCPFTG $(n=20)$.

At 2 months after the initiation of the animal model, $85 \%$ of each squamous cell carcinoma tumor was removed in the animals of the second group, and the $0.4-0.6-\mathrm{cm}$ diameter wound was closed with knotted 7.0 atraumatic sutures (Ethicon, Inc.; Johnson \& Johnson).

In the animals of the third group, at 2 months from the beginning of modeling, $85 \%$ of each squamous cell carcinoma tumor was also removed, and MCPFTG was introduced into the $0.4-0.6-\mathrm{cm}$ diameter wound. Initially, the wound was covered with PGF gel that contained CPtCS (internal layer). Next, a solution containing $1.0 \%$ calcium chloride and $52 \mathrm{U} / \mathrm{ml}$ thrombin (MilliporeSigma) diluted in saline solution was added to the PGF gel. While merging, a glutinous mass was formed between these two components, and it became transparent within 5-6 sec. PGF that was seeded with CultiSpher-S + BMSCs was placed on top of the internal layer, while a solution containing $1.0 \%$ calcium chloride and $52 \mathrm{U} / \mathrm{ml}$ thrombin was used for solidifying the external layer.

All surgical procedures were conducted under general anesthesia using intraperitoneal injection of pentobarbital sodium $(30 \mathrm{mg} / \mathrm{kg})$.

Postoperative care. Animals were housed in standard laboratory conditions under normal day and night cycles with provision of pelleted rodent diet and water ad libitum. During the postoperative period, the rate of wound healing was determined using the planimetric method. 
On days $30,40,60,90,120$ and 150 after surgery, the rats were euthanized with a lethal dose $(200 \mathrm{mg} / \mathrm{kg})$ of sodium thiopental solution via intraperitoneal injection. At necropsy, full thickness skin samples covering the jaw and neck, including subcutaneous tissue and tissue of the tongue with a tumor were excised for histopathological studies.

Scanning electron microscopy. Scanning electron microscopy was performed as previously described (17). Briefly, the collected wound tissues were immersed in a fixative solution containing $2.5 \%$ glutaraldehyde and $4 \%$ paraformaldehyde in $0.1 \mathrm{M}$ of phosphate buffer. After fixation, the samples were dehydrated in 30, 50, 70, 80 and $90 \%$ ethanol (for 10 min each) and twice in $95 \%$ ethanol (20 min each) in succession at room temperature. The samples were then immersed in a mixture of $95 \%$ ethanol and isoamyl acetate (1:1) for $10 \mathrm{~min}$, and in pure isoamyl acetate for $15 \mathrm{~min}$. After removing isoamyl acetate, the samples were dried with a Tousimis Samdri-780 Critical Point Dryer (Tousimis Research Corporation). Next, the tissues were sputter coated lightly with gold and imaged on a JEOL JSM-65 10 LW scanning electron microscope (JEOL, Ltd.).

Energy dispersive spectroscopy. Energy dispersive spectroscopy was used to determine the quantity of CPtCS in the MCPFTG before and after it was applied to the wound. For energy dispersive analysis of the results of scanning electron microscopy, the analytical AztecEnergy-EDS software (Oxford Instruments, Inc.) and the X-MaxN SDD detector (Oxford Instruments, Inc.) were used.

Method of determination of wound healing speed. For an objective assessment of wound healing speed, and to acknowledge the changes in size of the wound within a certain period of time, the method of planimetry was used in all animals. For this purpose, a sheet of cellophane sterilized in an autoclave was applied to the wound, on which the contours of the wound were outlined with ink. Next, the cellophane with the outlined contour was placed on a paper denoting $\mathrm{mm}$, and the area of the wound was determined by counting the number of $\mathrm{mm}^{2}$ inside the contour. The percentage of daily wound reduction was determined by using the following formula: $[(S-S n) \times 100] /(S \times t)$, where $\mathrm{S}$ is the wound area during any subsequent measurement, $\mathrm{Sn}$ is the wound area during the current measurement and $t$ is the number of days between the measurements.

Tumor volume determination, and histopathological and immunohistochemical analyses. After establishing a rat model of squamous cell carcinoma of the tongue, the tumor volume was measured during the autopsy after collection of material for histopathological analysis at various time points. The tumor volume was calculated according to the formula: Volume $=$ length $\mathrm{x}$ width $\mathrm{x}$ height $\mathrm{x}(\pi / 6)$.

For histopathological studies, the tongue tumor and its surrounding tissues were fixed in $10 \%$ neutral buffered formalin for $24 \mathrm{~h}$ at room temperature, embedded in paraffin and cut into 5- $\mu \mathrm{m}$ thick slices. After deparaffinization and rehydration, the tissues were stained with hematoxylin and eosin (Leica Biosystems Newcastle Ltd.), and Masson's trichrome (Bio-Optica Ltd.), at room temperature for $35 \mathrm{~min}$ according the manufacturers' protocols. Immunostaining with anti-p63 (incubation time, 20 min; clone 7JUL; dilution 1:25; cat. no. PA0103; Leica Biosystems Newcastle Ltd.), anti-Ki67 (incubation time, $20 \mathrm{~min}$; clone MM1; dilution 1:200; cat. no. PA0118; Leica Biosystems Newcastle Ltd.), anti-cyclin D1 (incubation time, $20 \mathrm{~min}$; clone EP12; dilution 1:50; cat. no.PA0046; Leica Biosystems Newcastle Ltd.) and anti-E cadherin (incubation time, $20 \mathrm{~min}$; clone 36B5; dilution 1:25; cat. no. PA0387; Leica Biosystems Newcastle Ltd.) antibodies was performed with the manual staining method using the Novolink DAB Polymer Detection system (incubation time, 20 min; cat no. RE7260-CE; Leica Biosystems Newcastle Ltd.) according to the manufacturer's recommendations. Endogenous peroxidase activity was neutralized using the Peroxidase Block reagent [3-4\% (v/v) hydrogen peroxide; Novolink DAB Polymer Detection system; cat. no. RE7260-CE; Leica Biosystems Newcastle Ltd]. To reduce non-specific binding of primary antibody Novocastra Protein Block reagent was used $(0.4 \%$ casein in phosphate-buffered saline, with stabilizers, surfactant and $0.2 \%$ Bronidox L as a preservative; Novolink DAB Polymer Detection system). As the secondary antibody, rabbit anti-mouse $\operatorname{IgG}(<10 \mu \mathrm{g} / \mathrm{ml})$ in $10 \%(\mathrm{v} / \mathrm{v})$ animal serum in Tris-buffered saline/0.1\% ProClin ${ }^{\text {TM }} 950$ was used (Novolink DAB Polymer Detection system). All reactions for immunohistochemistry were performed at room temperature. The samples were next observed under a light microscope. For image capture, the LAS V4.0 (Leica Microsystems Ltd.) software was used. Images than was analyzed using ImageJ v1.51 (National Institutes of Health). Percentage of positive nuclei were counted using the ImmunoRatio macros plugin for ImageJ. Intensity of cytoplasmic staining was evaluated by a 4-tier visual scoring method $(0$, no staining; $1+$, weak; $2+$, moderate; and $3+$, strong ).

Statistical analysis. Statistical analysis was performed using GraphPad Prism 9.0 software (GraphPad Software, Inc.). Tumor growth was calculated and compared between groups for each time point, and statistical analysis was assessed with one-way ANOVA followed by Tukey's post hoc test. All experiments were repeated at least three times. $\mathrm{P}<0.05$ was considered to indicate a statistically significant difference.

\section{Results}

At 2 months after the creation of a model of squamous cell carcinoma in 60 rats on the surface of the tongue, a well-defined carcinoma in situ was visible, the presence of which was confirmed by histopathological and immunohistochemical methods. (Fig. 1).

Loss of epithelial cell polarity, nuclear polymorphism and hyperchromatism were evident in the tissue of the tongue. Abnormal keratinization (dyskeratosis) of individual cells, cytological disorganization and abnormal mitosis without mucous membrane invasion were also observed. Inflammatory cells were detected in the connective tissue. Dysplasia, as well as hyperplasia and carcinoma in situ, were identified at different time points in all groups of animals. High expression of cyclin D1 and low expression of E-cadherin were revealed by immunohistochemistry. At a later period, 3.5-4 months after initiation of the model, a well differentiated squamous cell carcinoma with signs of keratin formation was observed.

Over the entire 5-month period, the size of the tumors (Fig. 2) in the animals of groups I and II were significantly 


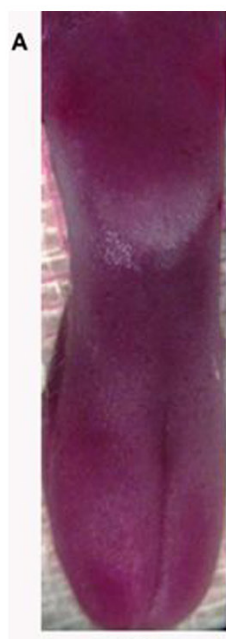

a

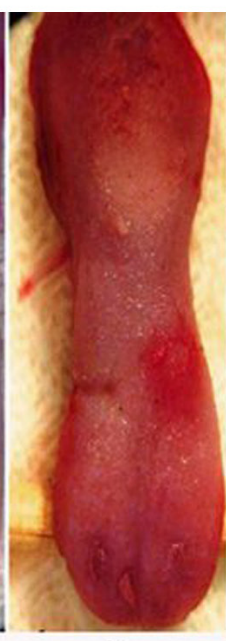

b

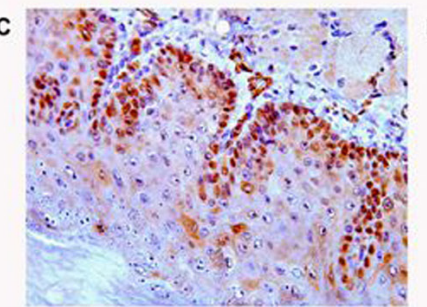

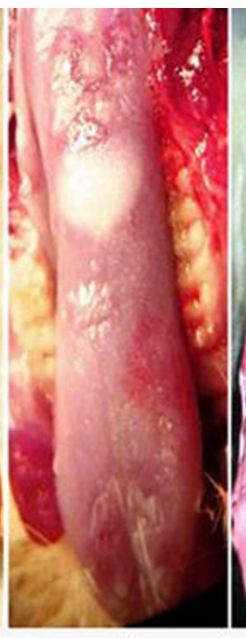

c

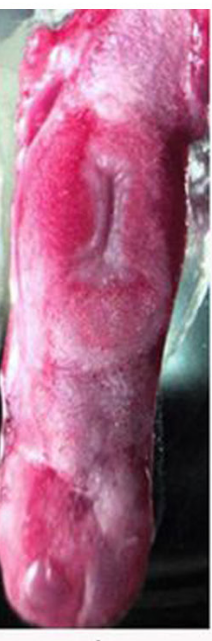

d
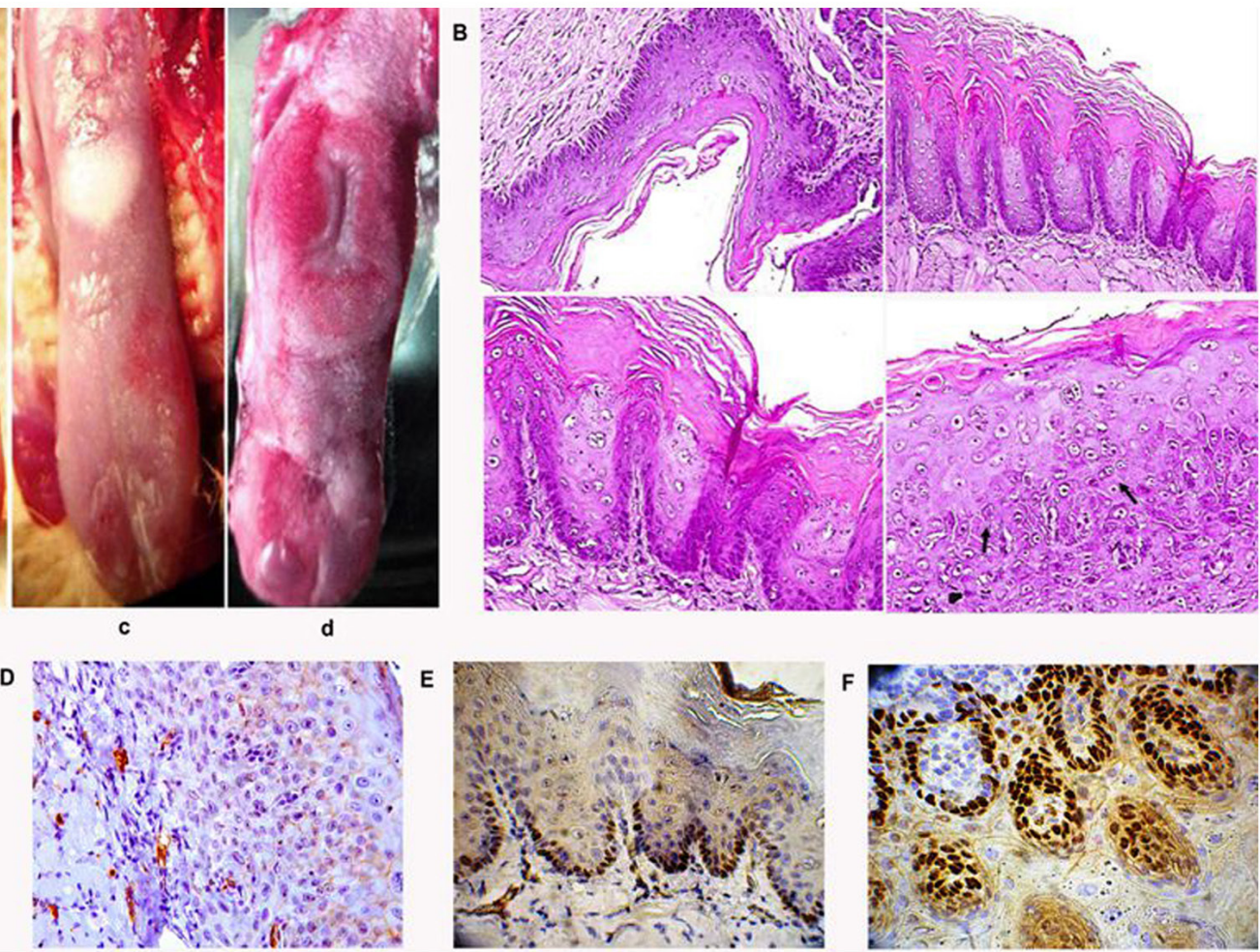

Figure 1. Modeling of tongue squamous cell carcinoma in rats. (A) Rat tongue lesions induced by 4-nitroquinoline 1-oxide: (a) Normal tongue, and (b) 1, (c) 1.5 and (d) 2 months from the initiation of squamous cell carcinoma modeling. (B) Carcinoma in situ (black arrows) after an observation period of 2 months (hematoxylin and eosin staining; magnification, x400). (C) High expression of cyclin D1 (magnification, x400). (D) Low expression of E-cadherin (magnification, $\mathrm{x} 400$ ). (E) High expression of Ki-67 (magnification, x400). (F) High expression of p63 (magnification, x400).

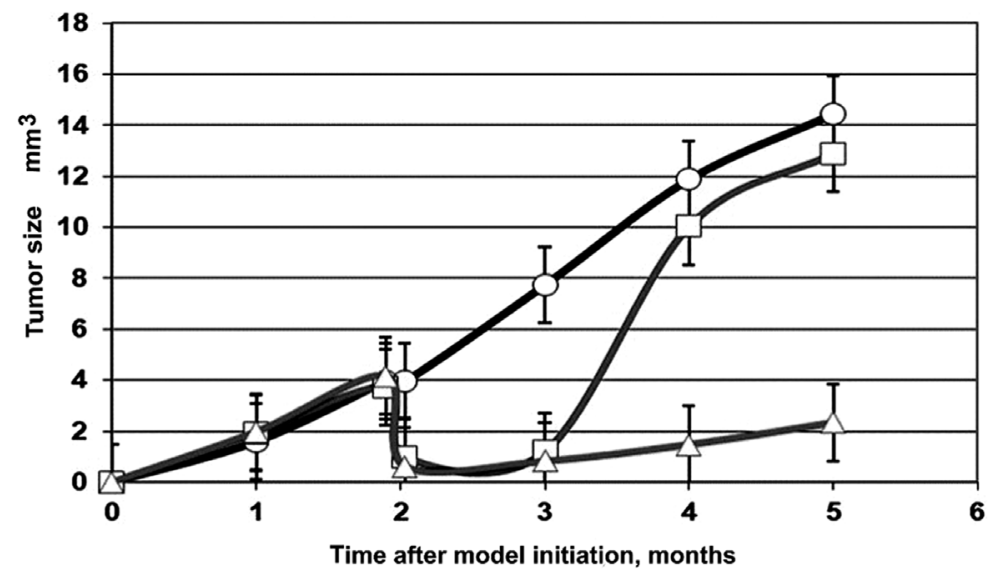

$\rightarrow$ Group I $\Longrightarrow$ Group II $\Longrightarrow$ Group III

Figure 2. Influence of multicomponent fibrin-based gel on the size of the tongue tumor that was induced by 4-nitroquinoline 1-oxide in rats of groups I, II and III.

different [mean difference, $0.7 ; 95 \%$ confidence interval (CI), $0.1558-1.244 ; \mathrm{P}=0.0097]$. The difference between the size of the tumors in the animals of groups II and III was also significant (mean difference, 11.34; 95\% CI, 10.80-11.88; $\mathrm{P}<0.0001$ ). The difference between the size of the tumors in the animals of groups I and III was also significant (mean difference, 12.04; 95\% CI, 11.50-12.58; P<0.0001).

Compared with that of the first group, the rate of tumor growth after surgery was slightly suppressed for 24 days in the animals of the second group. However, after 30-35 days, the tumor began to progress rapidly. It should be noted that in animals of the first and second groups, a decrease in body weight was observed (Fig. 3).

Histological examination of animals of the second group identified 14 (70\%) cases of highly differentiated squamous cell carcinoma of the tongue. In the rest of the animals of this group, epithelial dysplasia with varying degrees of atypia was observed at different time points. Immunohistochemical analysis revealed high expression of cyclin D1 and low expression of E-cadherin. High expression of Ki-67 and p63 was also observed (Fig. 4A-G). 


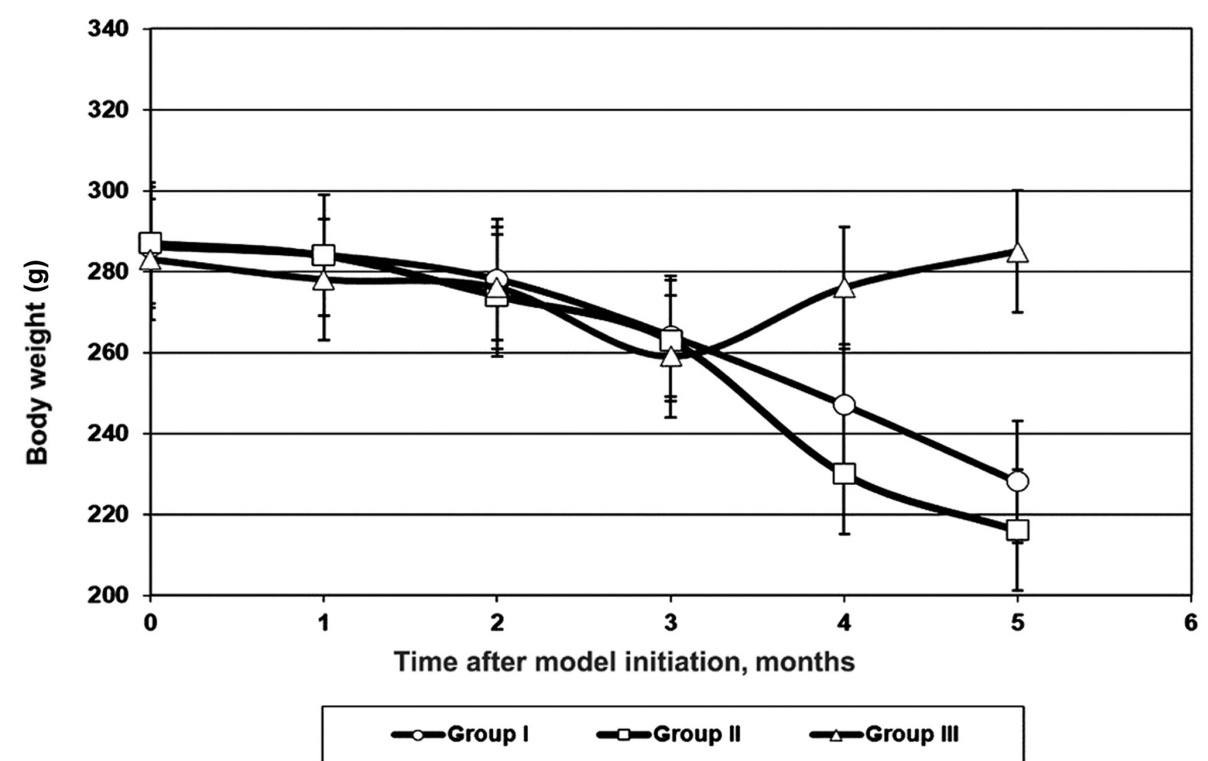

Figure 3. Body weight changes in animals of groups I, II and III.
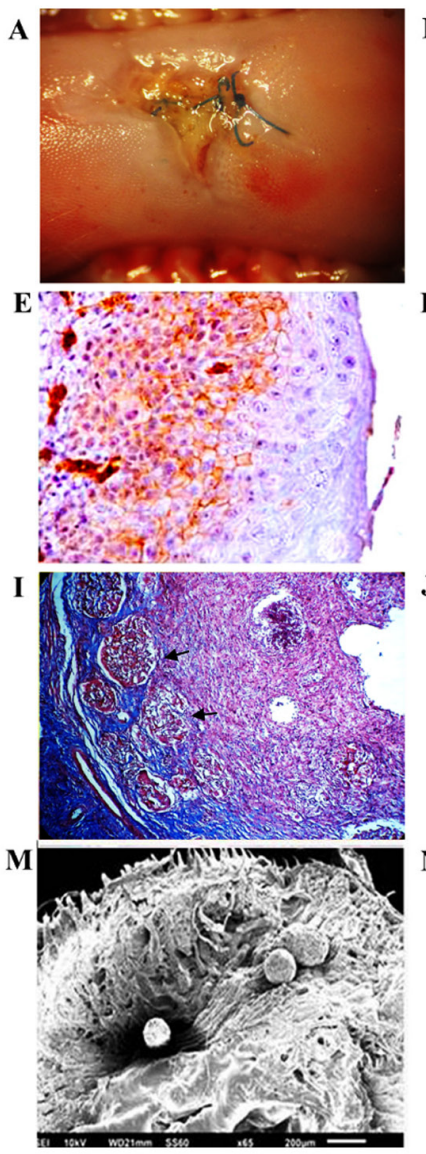
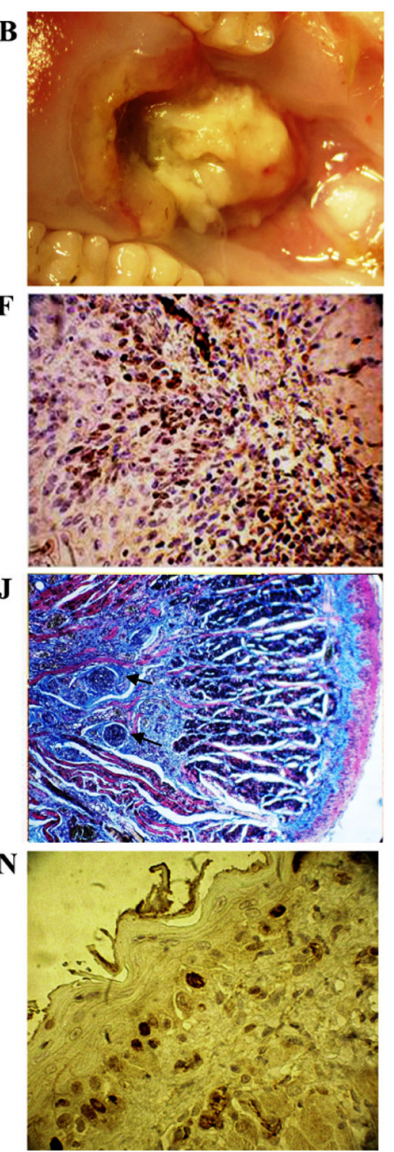
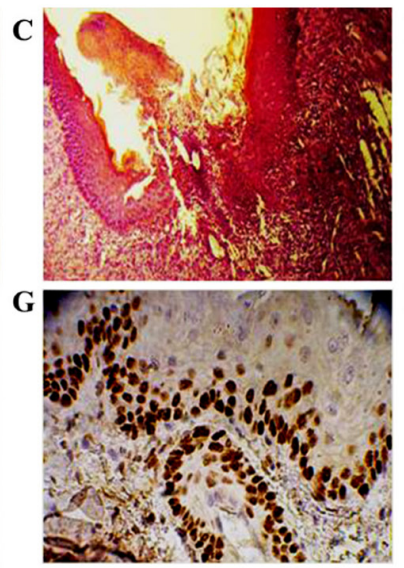

$\mathbf{K}$
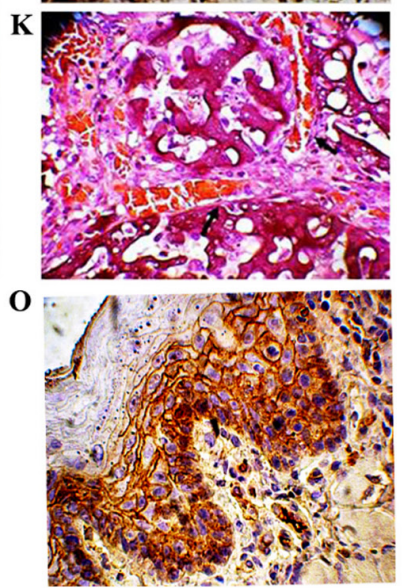
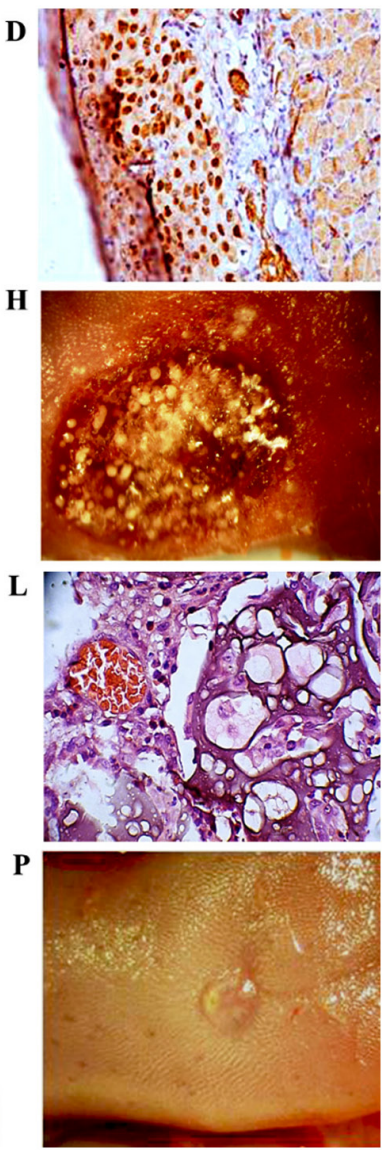

Figure 4. Process of wound healing after $85 \%$ resection of tongue squamous cell carcinoma and treatment with MCPFTG. (A-G) Group II, 4-NQO + surgery: (A) Non-healing wound of the tongue after $85 \%$ resection of squamous cell carcinoma (observation period, 20 days). (B) Tumor progression (observation period, 25-30 days). (C) Non-healing wound of the tongue. Hematoxylin and eosin staining; magnification, x200 (observation period, 15 days). (D) High expression of cyclin D1; magnification, $x 400$ (observation period, 30 days). (E) Low expression of E-cadherin; magnification, $\mathrm{x} 400$ (observation period, 30 days). (F) High expression of Ki-67; magnification, $x 400$ (observation period, 30 days). (G) High expression of p63; magnification x400 (observation period, 30 days). (H-P) Group III, 4-NQO + surgery + MCPFTG: (H) After $85 \%$ resection of squamous cell carcinoma, the wound of the tongue was covered with MCPFTG. (I) Wound bed with incorporated microcarriers (CultiSpher-S; black arrows). Masson's trichrome staining; magnification, x200 (observation period, 2 days). (J) Section of re-epithelialized wound bed with incorporated microcarriers. Residual CultiSpher-S were detected in the wound (black arrows). Masson's trichrome; magnification, x200 (observation period, 45 days). (K and L) Presence of connective tissue and neo-angiogenesis around the CultiSpher-S (black arrows). Hematoxylin and eosin staining; magnification, $\mathrm{x} 400$ (observation period, 20 days). (M) Scanning electron microscopy. CultiSpher-S was placed in the wound. (N) Low expression of cyclin D1; magnification, x400 (observation period, 3 months). (O) High expression of E-cadherin; magnification, x400 (observation period, 3 months). (P) Re-epithelialization of the wound after removal of the tumor and use of MCPFTG; observation period, $20-25$ days. 
In the present study, the humane endpoints for the size of the carcinoma of the tongue were set in the range of 12-14 $\mathrm{mm}^{3}$. Macroscopic examination of the liver, lungs and kidneys revealed no metastasis. Wound healing analysis showed that the majority of animals in the second group had non-healing tongue wounds within 3 months after tumor removal.

In most animals of the third group, the wounds healed within 20-25 days after tumor removal and MCPFTG application. Suppression of the tumor growth throughout 150 days without tumor recurrence was evident in the all animals treated with MCPFTG. Macroscopic examination of the liver, lungs and kidneys revealed no metastasis.

The results of the research showed that MCPFTG had formed a three-dimensional structure that filled the entire wound, and the surface area of contact of cisplatin with the remaining tumor cells was increased. Immunohistochemical analysis revealed low and moderate expression of cyclin D1, as well as high expression of E-cadherin throughout the whole observation period (Fig. 4H-P).

\section{Discussion}

Fibrinogen/thrombin gel has long been widely used in surgical practice; it is also used as a wound dressing for the closure of wounds (18). Fibrinogen/thrombin gel are important in tissue engineering (19). However, it has been reported that fibrinogen affects tumor growth and metastasis in vivo $(20,21)$. Additionally, three-dimensional fibrin gel promotes the selection and growth of tumorigenic cells (22). A previous study reported that in situ condensation of an anticancer drug into fibrin gel enables effective inhibition of tumor cell growth (23). The efficacy of the clinical application of human fibrinogen-thrombin patch $\left(\mathrm{TachoSil}^{\circledR}\right)$ in upper gastrointestinal cancer surgery has been demonstrated in another study (24). Currently, the bioresorbable polymer carriers of fibrinogen-based micro- and nanostructures are effectively used for creating targeted drug delivery systems. Fibrinogen has successfully been used as a 5-fluoroaracil carrier for cancer drug delivery applications (25). It has also been reported that fibrin glue enhances antitumor performance in models of subcutaneous and abdominal metastasis of murine colorectal cancer (26).

The present study aimed to develop a gel that, over a period of time, could release anticancer drugs locally to prevent recurrence and concomitantly accelerate wound healing.

The local drug delivery system based on MCPFTG that was developed in the present study has demonstrated efficacy in treating squamous cell carcinoma of the tongue in rats. Importantly, in $80 \%$ of the animals that were treated with MCPFTG, suppression of tumor growth was notable for 150 days without tumor recurrence. Additionally, in the majority of animals, at 45-50 days from tumor resection and introduction of MCPFTG into the wound, complete epithelialization of the wound was observed.

Scanning electron microscopy demonstrated that CPtCS was placed in the MCPFTG without being damaged, and was evenly distributed both on the surface and within the gel. The platinum cisplatin nanoparticles with a size of 10.7-11.2 nm had a cylindrical shape. Micro Energy-dispersive X-ray spectroscopy analysis showed that MCPFTG contained $29.1 \mu \mathrm{g}$ platinum nanoparticles. After placement in the wound, the quantity of platinum nanoparticles did not change for 5 months, and consequently they exhibited a long-term effect on residual tumor cells.

Overall, the current study showed that MCPFTG exerted an inhibitory effect on the growth of tongue squamous cell carcinoma. MCPFTG reduced the toxicity of cisplatin, and improved its antitumor activity by inhibiting the migration, invasion and proliferation of squamous cell carcinoma in rats.

CultiSpher-S microcarrier can function as a tissue scaffold and be beneficial as a temporary matrix for wound healing. A previous study reported similar results (27); however, in the current study, the freeze-dried BMSCs that were attached to the microcarrier significantly accelerated the re-epithelialization of the wound. Since CultiSpher-S microcarrier promotes in vitro cell attachment and migration, it was hypothesized that CultiSpher-S microcarrier can become an in vivo trap for residual tumor cells after it has been removed. The porous structure of the microcarrier allows the attachment and migration of tumor cells both on the surface and the inner structure of the microcarrier, and the cisplatin-loaded microcarrier is expected to destroy tumor cells.

We hypothesized that the internal layer of MCPFTG that directly contacted the surface of the wound could release cisplatin locally over a certain period of time, and destroy any residual tumor cells. The external layer of MCPFTG consisted of Cultispher-S microcarrier and freeze-dried BMSCs. Cultispher-S filled the residual wound cavities creating a 3-dimensional structure, and freeze-dried paracrine factors of BMSCs enhanced the wound regeneration process. Additionally, the possibility of using freeze-dried BMSCs for the treatment of non-healing wounds has been discussed in detail in our previous studies articles. The first study used a radiation wound model in rats. It was found that freeze-dried BMSCs retained their unique paracrine factors and improved clinical healing and re-epithelialization of non-healing wounds (28). Stem cell therapy for the treatment of non-healing chronic wounds has demonstrated positive therapeutic potential in a number of preclinical and clinical studies $(29,30)$. Cells are known to be potential sources of paracrine factors, and, as previously indicated, these paracrine bioactive factors are associated with the therapeutic effects of stem cells in chronic wound treatment (31). As previously reported, mesenchymal stem cell (MSC)-conditioned media contains keratinocyte growth factor, epidermal growth factor, VEGF- $\alpha$, insulin-like growth factor 1, stromal cell-derived factor 1 , erythropoietin, and macrophage inflammatory proteins $1 \alpha$ and $1 \beta$ (28). MSCs may accelerate the wound-healing process via a paracrine mechanism $(32,33)$. MSCs are also able to secrete paracrine angiogenesis-enhancing factors such as granulocyte colony-stimulating factor, VEGF, hepatocyte growth factor, interleukin 6, monocyte chemotactic protein-1 and TGF $\beta 1(34,35)$. However, there are no conclusive answers with regard to the mechanism by which MSCs exert positive effects (31).

Our second previously published study concerned the reconstruction of defects within the mandible using 
autogenous bone and decellularized bovine bone grafts with freeze-dried BMSC paracrine factors. The study consisted of one man and three women (age range, 38-55 years) who underwent surgery for a primary tumor of the mandible between January 2008 and December 2015 in the Cancer Research Center of Tbilisi (Tbilisi, Georgia) (36). Initial clinical investigations revealed that a biologically active bone graft seeded with freeze-dried BMSC paracrine factors could be applied for reconstructing large mandibular bone defects after tumor resection. The biologically active bone graft contained type III collagen, glycoproteins and a number of different growth factors, including basic fibroblast growth factor, epidermal growth factor, VEGF, keratinocyte growth factor, TGF $\alpha$ and $\beta$, PDGF, nerve growth factor and hepatocyte growth factor.

It has been reported that MSCs are able to migrate towards the tumor regardless of the type of tumor. This process of migration is mediated via a paracrine signaling loop between tumor microenvironment chemoattractants and corresponding receptor expression in MSCs (37). There are several reports demonstrating successful treatment of various types of tumor by using MSCs as carriers for anticancer drug delivery (38-42). As aforementioned, freeze-dried BMSCs are able to retain $>80 \%$ of paracrine factors, including VEGF-1, epidermal growth factor, insulin-like growth factor 1, hepatocyte growth factor, monocyte chemoattractant protein-1, angiopoietin-1, stromal cell-derived factor-1, erythropoietin and keratinocyte growth factor (28). It could be suggested that freeze-dried BMSC paracrine factors can attract residual tumor cells and enable their attachment to the surface of the CultiSpher-S microcarrier. However, this hypothesis requires further research for verification.

The mechanism by which MCPFTG suppresses the growth of tumor cells remains unclear. Further studies are required to understand the mechanism of tumor growth suppression mediated by MCPFTG. A limitation of the present study is associated with the use of paracrine factors of bone marrow stem cells, which require more detailed study into their role in tumor treatment.

We are currently conducting western blotting studies on the expression of several related proteins and hope that these results will be reflected in future publications.

In conclusion, the MCPFTG-based local drug delivery system has been shown to be effective in suppressing tumor growth and preventing recurrence. In the present study, MCPFTG reduced the toxicity of cisplatin and improved its antitumor activity. In addition, freeze-dried BMSC paracrine factors presented in the MCPFTG enhanced the wound healing processes after tumor removal. Thus, the present study suggests novel opportunities for the development of a multifunctional drug delivery system for the treatment of squamous cell carcinoma.

\section{Acknowledgements}

Not applicable.

\section{Funding}

No funding was received.

\section{Availability of data and materials}

The datasets used and/or analyzed during the current study are available from the corresponding author on reasonable request.

\section{Authors' contributions}

The authors confirm contribution to the paper as follows: MZK and TP performed experimental studies, data analysis and participated in the preparation of the manuscript. NK was responsible for the study concept. KM and ZV processed experimental data and performed data interpretation for the study. MJ and KG performed histological and immunohistochemical studies, and the statistical analysis. LK and DC processed experimental data and confirm the authenticity of all the raw data. ZK conceived and planned the experiments, performed the analysis and the prepared of the manuscript. All authors read and approved the final version of the manuscript.

\section{Ethics approval and consent to participate}

The housing and handling of the rats followed guidelines established by the Animal Care and Use Committee of Tbilisi State Medical University (Tbilisi, Georgia). Animals were monitored daily for infection and other illnesses by trained animal technicians. All individuals who handled rats were registered to protocols at Tbilisi State Medical University. All animal protocols were approved by the Institutional Review Board of Tbilisi State Medical University (approval no. 812).

\section{Patient consent for publication}

Not applicable.

\section{Competing interests}

The authors declare that they have no competing interests.

\section{References}

1. Torre LA, Bray F, Siegel RL, Ferlay J, Lortet-Tieulent J and Jemal A: Global cancer statistics, 2012. CA Cancer J Clin 65: 87-108, 2015.

2. Johnson NW, Jayasekara P and Amarasinghe AA: Squamous cell carcinoma and precursor lesions of the oral cavity: Epidemiology and aetiology. Periodontol 2000 57: 19-37, 2011.

3. Mahvi DA, Liu R, Grinstaff MW, Colson YL and Raut CP: Local Cancer Recurrence: The Realities, Challenges, and Opportunities for New Therapies. CA Cancer J Clin 68: 488-505, 2018.

4. Oliver RJ, Clarkson JE, Conway DI, Glenny A, Macluskey M, Pavitt S, Sloan P and Worthington HV; CSROC Expert Panel: Interventions for the treatment of oral and oropharyngeal cancers: Surgical treatment. Cochrane Database Syst Rev 4: CD006205, 2007.

5. Bulsara VM, Worthington HV, Glenny AM, Clarkson JE, Conway DI and Macluskey M: Interventions for the treatment of oral and oropharyngeal cancers: Surgical treatment. Cochrane Database Syst Rev 12: CD006205, 2018.

6. Sun LM, Leung SW, Su CY and Wang CJ: The relapse patterns and outcome of postoperative recurrent tongue cancer. J Oral Maxillofac Surg 55: 827-831, 1997.

7. Kishi N, Imai Y, Kanayama N, Hirata T, Kawaguchi Y, Konishi K, Nishiyama K and Teshima T: Recurrence Patterns of Postoperative Radiation Therapy for Patients with Head and Neck Squamous Cell Carcinoma. Int J Radiat Oncol Biol Phys 96: E389-E390, 2016. 
8. Liao CT, Chang JT, Wang HM, Ng SH, Hsueh C, Lee LY, Lin $\mathrm{CH}$, Chen IH, Huang SF, Cheng AJ, et al: Salvage therapy in relapsed squamous cell carcinoma of the oral cavity: How and when? Cancer 112: 94-103, 2008.

9. Rowe DE, Carroll RJ and Day CL Jr: Prognostic factors for local recurrence, metastasis, and survival rates in squamous cell carcinoma of the skin, ear, and lip. Implications for treatment modality selection. J Am Acad Dermatol 26: 976-990, 1992.

10. Larsen SR, Johansen J, Sørensen JA and Krogdahl A: The prognostic significance of histological features in oral squamous cell carcinoma. J Oral Pathol Med 38: 657-662, 2009.

11. Woolgar JA and Triantafyllou A: A histopathological appraisal of surgical margins in oral and oropharyngeal cancer resection specimens. Oral Oncol 41: 1034-1043, 2005.

12. Zhang G, Wang X, Wang Z, Zhang J and Suggs L: A PEGylated fibrin patch for mesenchymal stem cell delivery. Tissue Eng 12: 9-19, 2006

13. Kakabadze Z, Kipshidze N, Mardaleishvili K, Chutkerashvili G, Chelishvili I, Harders A, Loladze G, Shatirishvili G, Kipshidze N, Chakhunashvili D, et al: Phase 1 Trial of Autologous Bone Marrow Stem Cell Transplantation in Patients with Spinal Cord Injury. Stem Cells Int 2016: 6768274, 2016

14. Sagheer SH, Whitaker-Menezes D, Han JYS, Curry JM, Martinez-Outschoorn U and Philp NJ: 4NQO induced carcinogenesis: A mouse model for oral squamous cell carcinoma. Methods Cell Biol 163: 93-111, 2021.

15. Hawkins BL, Heniford BW, Ackermann DM, Leonberger M, Martinez SA and Hendler FJ: 4NQO carcinogenesis: A mouse model of oral cavity squamous cell carcinoma. Head Neck 16: 424-432, 1994

16. El-Rouby DH: Histological and immunohistochemical evaluation of the chemopreventive role of lycopene in tongue carcinogenesis induced by 4-nitroquinoline-1-oxide. Arch Oral Biol 56: 664-671, 2011

17. Kakabadze Z, Kakabadze A, Chakhunashvili D, Karalashvili L, Berishvili E, Sharma Y and Gupta S: Decellularized human placenta supports hepatic tissue and allows rescue in acute liver failure. Hepatology 67: 1956-1969, 2018.

18. Rothwell SW, Sawyer E, Dorsey J, Flournoy WS, Settle T, Simpson D, Cadd G, Janmey P, White C and Szabo KA: Wound healing and the immune response in swine treated with a hemostatic bandage composed of salmon thrombin and fibrinogen. J Mater Sci Mater Med 20: 2155-2166, 2009.

19. Ahmed TA, Dare EV and Hincke M: Fibrin: A versatile scaffold for tissue engineering applications. Tissue Eng Part B Rev 14: 199-215, 2008.

20. Costantini V and Zacharski LR: The role of fibrin in tumor metastasis. Cancer Metastasis Rev 11: 283-290, 1992

21. Staton CA, Brown NJ and Lewis CE: The role of fibrinogen and related fragments in tumour angiogenesis and metastasis. Expert Opin Biol Ther 3: 1105-1120, 2003.

22. Liu J, Tan Y, Zhang H, Zhang Y, Xu P, Chen J, Poh YC, Tang K, Wang $\mathrm{N}$ and Huang B: Soft fibrin gels promote selection and growth of tumorigenic cells. Nat Mater 11: 734-741, 2012.

23. Kuwahara M, Fujita H, Kataoka Y, Nakajima Y, Yamada M and Sugimoto N: In situ condensation of an anti-cancer drug into fibrin gel enabling effective inhibition of tumor cell growth. Chem Commun (Camb) 55: 11679-11682, 2019.

24. MaranoLandDiMartinoN:Efficacy ofHumanFibrinogen-Thrombin Patch (TachoSil) Clinical Application in Upper Gastrointestinal Cancer Surgery. J Invest Surg 29: 352-358, 2016.

25. Rejinold NS, Muthunarayanan M, Chennazhi KP, Nair SV and Jayakumar R: 5-fluorouracil loaded fibrinogen nanoparticles for cancer drug delivery applications. Int J Biol Macromol 48: 98-105, 2011

26. Hu Y, Yu T, Liu X, He Y, Deng L, Guo J, Hua Y, Luo T and Gao X Improved anti-tumor efficacy via combination of oxaliplatin and fibrin glue in colorectal cancer. Oncotarget 9: 2515-2526, 2017.

27. Lönnqvist S, Rakar J, Briheim K and Kratz G: Biodegradable Gelatin Microcarriers Facilitate Re-Epithelialization of Human Cutaneous Wounds - An In Vitro Study in Human Skin. PLoS One 10: e0128093, 2015.
28. Kakabadze Z, Chakhunashvili D, Gogilashvili K, Ediberidze K, Chakhunashvili K, Kalandarishvili K and Karalashvili L: Bone Marrow Stem Cell and Decellularized Human Amniotic Membrane for the Treatment of Nonhealing Wound After Radiation Therapy. Exp Clin Transplant 17 (Suppl 1): 92-98, 2019.

29. Marfia G, Navone SE, Di Vito C, Ughi N, Tabano S, Miozzo M, Tremolada C, Bolla G, Crotti C, Ingegnoli F, et al: Mesenchymal stem cells: Potential for therapy and treatment of chronic non-healing skin wounds. Organogenesis 11: 183-206, 2015.

30. Asanuma H, Meldrum DR and Meldrum KK: Therapeutic applications of mesenchymal stem cells to repair kidney injury. J Urol 184: 26-33, 2010

31. Burdon TJ, Paul A, Noiseux N, Prakash S and Shum-Tim D: Bone marrow stem cell derived paracrine factors for regenerative medicine: Current perspectives and therapeutic potential. Bone Marrow Res 2011: 207326, 2011.

32. Chen L, Tredget EE, Wu PY and Wu Y: Paracrine factors of mesenchymal stem cells recruit macrophages and endothelial lineage cells and enhance wound healing. PLoS One 3: e1886, 2008.

33. Walter MN, Wright KT, Fuller HR, MacNeil S and Johnson WE: Mesenchymal stem cell-conditioned medium accelerates skin wound healing: An in vitro study of fibroblast and keratinocyte scratch assays. Exp Cell Res 316: 1271-1281, 2010.

34. Kwon HM, Hur SM, Park KY, Kim CK, Kim YM, Kim HS, Shin HC, Won MH, Ha KS, Kwon YG, et al: Multiple paracrine factors secreted by mesenchymal stem cells contribute to angiogenesis. Vascul Pharmacol 63: 19-28, 2014.

35. Baraniak PR and McDevitt TC: Stem cell paracrine actions and tissue regeneration. Regen Med 5: 121-143, 2010.

36. Kakabadze A, Mardaleishvili K, Loladze G, Karalashvili L, Chutkerashvili G, Chakhunashvili D and Kakabadze Z: Reconstruction of mandibular defects with autogenous bone and decellularized bovine bone grafts with freeze-dried bone marrow stem cell paracrine factors. Oncol Lett 13: 1811-1818, 2017.

37. Chan JK and Lam PY: Human mesenchymal stem cells and their paracrine factors for the treatment of brain tumors. Cancer Gene Ther 20: 539-543, 2013

38. Sasportas LS, Kasmieh R, Wakimoto H, Hingtgen S, van de Water JA, Mohapatra G, Figueiredo JL, Martuza RL, Weissleder R and Shah K: Assessment of therapeutic efficacy and fate of engineered human mesenchymal stem cells for cancer therapy. Proc Natl Acad Sci USA 106: 4822-4827, 2009.

39. Dwyer RM, Ryan J, Havelin RJ, Morris JC, Miller BW, Liu Z, Flavin R, O'Flatharta C, Foley MJ, Barrett HH, et al: Mesenchymal Stem Cell-mediated delivery of the sodium iodide symporter supports radionuclide imaging and treatment of breast cancer. Stem Cells 29: 1149-1157, 2011.

40. Li GC, Ye QH, Xue YH, Sun HJ, Zhou HJ, Ren N, Jia HL, Shi J, Wu JC, Dai C, et al: Human mesenchymal stem cells inhibit metastasis of a hepatocellular carcinoma model using the MHCC97-H cell line. Cancer Sci 101: 2546-2553, 2010

41. Zischek C, Niess H, Ischenko I, Conrad C, Huss R, Jauch KW, Nelson PJ and Bruns C: Targeting tumor stroma using engineered mesenchymal stem cells reduces the growth of pancreatic carcinoma. Ann Surg 250: 747-753, 2009.

42. Khakoo AY, Pati S, Anderson SA, Reid W, Elshal MF, Rovira II, Nguyen AT, Malide D, Combs CA, Hall G, et al: Human mesenchymal stem cells exert potent antitumorigenic effects in a model of Kaposi's sarcoma. J Exp Med 203: 1235-1247, 2006.

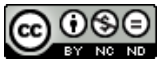

This work is licensed under a Creative Commons Attribution-NonCommercial-NoDerivatives 4.0 International (CC BY-NC-ND 4.0) License. 